\title{
Association between sucrose intake and acute coronary event risk and effect modification by lifestyle factors: Malmö Diet and Cancer Cohort Study
}

\author{
K. Warfa ${ }^{1}$, I. Drake ${ }^{1}$, P. Wallström ${ }^{2}$, G. Engström ${ }^{3}$ and E. Sonestedt ${ }^{1 *}$ \\ ${ }^{1}$ Diabetes and Cardiovascular Disease - Genetic Epidemiology, Department of Clinical Sciences Malmö, Lund University, \\ Jan Waldenströms gata 35, SE-20502 Malmö, Sweden \\ ${ }^{2}$ Nutritional Epidemiology, Department of Clinical Sciences Malmö, Lund University, Jan Waldenströms gata 35, SE-20502 \\ Malmö, Sweden \\ ${ }^{3}$ Cardiovascular Epidemiology, Department of Clinical Sciences Malmö, Lund University, Jan Waldenströms gata 35, \\ SE-20502 Malmö, Sweden
}

(Submitted 11 February 2016 - Final revision received 25 May 2016 - Accepted 2 August 2016 - First published online 24 October 2016)

\section{Abstract}

Previous studies have suggested that a high intake of sugar-sweetened beverages is positively associated with the risk of a coronary event. However, a few studies have examined the association between sucrose (the most common extrinsic sugar in Sweden) and incident coronary events. The objective of the present study was to examine the associations between sucrose intake and coronary event risk and to determine whether these associations are specific to certain subgroups of the population (i.e. according to physical activity, obesity status, educational level, alcohol consumption, smoking habits, intake of fat and intake of fruits and vegetables). We performed a prospective analysis on 26190 individuals ( $62 \%$ women) free from diabetes and without a history of CVD from the Swedish population-based Malmö Diet and Cancer cohort. Over an average of 17 years of follow-up (457 131 person-years), 2493 incident cases of coronary events were identified. Sucrose intake was obtained from an interview-based diet history method, including 7-d records of prepared meals and cold beverages and a 168-item diet questionnaire covering other foods. Participants who consumed $>15 \%$ of their energy intake (E\%) from sucrose showed a 37 (95\% CI 13 , $66) \%$ increased risk of a coronary event compared with the lowest sucrose consumers $(<5 \mathrm{E} \%)$ after adjusting for potential confounders. The association was not modified by the selected lifestyle factors. The results indicated that sucrose consumption higher than $15 \mathrm{E} \%(5 \%$ of this population) is associated with an increased risk of a coronary event.

Key words: Sucrose: CVD: Effect modification: Risk factors

CVD, including coronary events (CHD, i.e. myocardial infarction (MI)) and cerebrovascular disease (stroke), is the leading cause of death worldwide ${ }^{(1)}$. An unhealthy diet is one of the modifiable risk factors for CVD. The increased consumption of sugar-rich foods, particularly sugar-sweetened beverages, has been observed worldwide ${ }^{(2)}$. Sugars can be naturally occurring (intrinsic) in many foods such as vegetables, fruits and milk products or can be added to foods (extrinsic) ${ }^{(3)}$.

Excessive sugar consumption may reduce the intake of essential nutrients. Yet, the effect of added sugar on micronutrient dilution remains unknown ${ }^{(4-6)}$. High sugar intake may also increase energy intake, leading to unhealthy weight gain ${ }^{(7)}$, and thereby increasing the risk of non-communicable diseases such as type 2 diabetes and $\mathrm{CVD}^{(8)}$. Sugar may also directly contribute to CVD by influencing fat metabolism or inflammation. Recommendations on the maximum amount of energy from sugars vary in different regions and from different institutes - for example, the World Health Organization ${ }^{(9)}$ guidelines strongly recommend $<10 \%$ of energy from free sugars, whereas the US Institute of Medicine recommends $<25 \%{ }^{(10)}$ of energy from added sugar. The Nordic Nutrition Recommendations state that $<10 \%$ of energy should come from added sugar $^{(11)}$. A study among US adults indicated that the participants who consumed added sugar above the recommended WHO level (10\%) and below the US Institute of Medicine recommendation (25\%) had a $30 \%$ increased risk of cardiovascular mortality compared with individuals consuming $<10 \%{ }^{(8)}$.

Evidence of the long-term adverse effects of high sugar consumption on metabolic outcomes is inconsistent, and a few studies have examined the relationship between sugar consumption and CVD risk. According to systematic literature reviews, consuming sugar-sweetened beverages likely increases the risk of type 2 diabetes ${ }^{(12-14)}$, although the

Abbreviations: E\%, percentage of energy; HR, hazard ratio; MDC, Malmö Diet and Cancer.

* Corresponding author: E. Sonestedt, fax +46 403913 22, email emily.sonestedt@med.lu.se 
evidence for an association between the consumption of specific sugars (e.g. fructose or sucrose) and type 2 diabetes risk is not as conclusive ${ }^{(12)}$. The associations between the consumption of sugars or sugar-rich foods and CVD risk are not widely studied, and the evidence is inconclusive ${ }^{(12,15)}$. In addition, a few studies have investigated whether the association between consumption of sugars and risk of CVD is more pronounced in subgroups of the population ${ }^{(16)}$.

The most common sugar added to the diet (extrinsic sugar) in Sweden is sucrose, a disaccharide consisting of glucose and fructose $^{(3)}$. Sucrose can therefore also be utilised as a marker of added sugar in the Swedish diet. In the Malmö Diet and Cancer (MDC) cohort, men consuming $<10 \%$ of their energy from sucrose had a $14 \%$ lower risk of CVD (coronary events and ischaemic stroke) compared with individuals with higher intake levels. A similar trend (but not statistically significant) was observed for women ${ }^{(17)}$. In a more recent study within the MDC cohort, we did not observe a significantly increased risk of coronary events when comparing the highest and lowest quintiles of sucrose, although we observed a tendency among men $(\text { hazard ratio }(\mathrm{HR})=1 \cdot 16 ; 95 \% \mathrm{CI} 0 \cdot 97,1 \cdot 39)^{(18)}$. Therefore, the aim of the present study was to further examine the associations between sucrose intake and coronary event risk by evaluating the risk at more extreme, but still relevant, intake levels. For example, evaluating the association among individuals who consumed $<5$ percentage of energy (E\%) and $>15 \mathrm{E} \%$, and test whether the association is linear. We also used a longer follow-up period and examined whether these associations are specific to certain subgroups of the population according to lifestyle factors (i.e. physical activity, obesity status, educational level, alcohol consumption, smoking habits, fat intake, and fruit and vegetable intake). In particular, we examined whether moderate sucrose consumption (even below $10 \mathrm{E} \%$ level) is associated with an increased risk of coronary events in certain groups of individuals.

\section{Methods}

\section{Population and setting}

The present study was conducted in Sweden within the MDC cohort. The MDC study population (men and women aged between 44 and 73 years who lived in Malmö) was recruited between 1991 and 1996. The participants were recruited through either a passive method, in which research invitations were placed in public areas such as banks and post offices, or an active recruitment method, in which participants were invited through a personal letter sent to the population. If the participants did not respond to the first letter, second and third letters were also sent. Individuals who did not respond to the final letter received a follow-up phone call $^{(19)}$. Participants who had limited Swedish language skills or were mentally impaired were excluded from the study.

A baseline questionnaire was used to collect data on demographics, physical activity, educational level, smoking habits and alcohol consumption. Among the 74138 participants recruited into the MDC, 68905 were eligible, and 28098 subjects completed the baseline examination ${ }^{(19)}$. Mortality was lower among participants compared with non-participants during recruitment and for at least a few years after recruitment. However, the socio-economic structure and prevalence of smoking and obesity among MDC participants were comparable with the participants who took part in a mailed health survey during the same period ${ }^{(20)}$. In the present study, we excluded individuals with a history of CVD (coronary events or stroke from national registers) ( $n$ 820) and diabetes (self-reported diagnosis or use of diabetes medication) ( $n$ 870) at baseline, and those with missing data on smoking ( $n$ 12), BMI ( $n$ 42), leisure-time physical activity ( $n$ 191) and educational level ( $n$ 71), resulting in 26190 individuals available for analyses (online Supplementary Fig. S1). These participants were followed-up until the end point - that is, the occurrence of death, first cardiovascular event, emigration from Sweden or the end of follow-up (31 December 2013).

\section{Dietary data}

Dietary habits were measured using a modified diet history method combining a 7-d food diary, a semi-quantitative, 168item questionnaire and a diet history interview. The 7-d food diary was used to collect information on prepared meals and cold beverages, whereas the questionnaire collected information on frequencies (usually numbers per day, week or month) and portion sizes (from pictures) of foods not collected in the food diary ${ }^{(21)}$. During the food history interview, participants described their usual food preparation methods and portion sizes (using photographs) for the meals registered in the food diary. The Swedish Food Database was used to convert the food data collected from the modified diet history into total energy and nutrient intakes ${ }^{(22)}$. Sucrose was measured, or calculated, for all foods included in the database. The validity of the modified diet history method was examined against a reference method, including $18 \mathrm{~d}$ of weighed food records ${ }^{(22)}$. The validation correlation coefficient (Pearson's) of energyadjusted sucrose intake was relatively high in both males and females $(0.60 \text { and } 0.74 \text {, respectively })^{(23)}$.

In September 1994, there was a change in the coding of dietary data to reduce the interview time; however, this change did not influence the ranking of individuals according to dietary intake $^{(21)}$. The main exposure in the present study was sucrose intake, which was expressed as a percentage of non-alcohol energy ( $\mathrm{E} \%$ ), and the participants were categorised into five groups: $<5,5-7 \cdot 5,7 \cdot 5-10,10-15$ and $>15 \mathrm{E} \%$. The participants were also categorised into four groups according to their energy-adjusted intakes (using the residual model) of foods that contributed sucrose to the diet - that is, sweets, chocolates, sugar, jam/marmalade, fruit juice, sugar-sweetened beverages, and cakes and pastries.

\section{Assessment of other variables}

Weight and height were determined with shoes off, and the values were used to calculate BMI. Waist circumference was obtained after measuring the midpoint between the lowest rib margin and the iliac crest. Blood pressure was obtained while the participants rested, lying in a supine position. 
Data concerning smoking status (smokers, ex-smokers and non-smokers), leisure-time physical activity (sex-specific quintiles 1-5) and educational level (elementary or less, primary and secondary, upper secondary, further education without a degree and an university degree) were collected from the questionnaire. The level of leisure-time physical activity was determined using a questionnaire, in which the participants were asked to estimate the duration per week spent on seventeen different activities. The questions were adapted from the Minnesota Leisure Time Physical Activity Questionnaire ${ }^{(24)}$. The duration was multiplied by an intensity factor to create a leisure-time physical activity score. The participants were divided into sex-specific quintiles. Alcohol consumption was obtained from both the 7-d food diary and the questionnaire. Individuals who did not consume any alcohol within the $7 \mathrm{~d}$ or during the previous year in the questionnaire were categorised as zero consumers, and the remaining subjects were categorised into sex-specific quintiles according to intakes during the $7 \mathrm{~d}$. Individuals who reported a substantial dietary change in the past were classified as 'dietary changers, ${ }^{,(25)}$. Potential energy misreporters were identified by comparing reported energy intake with estimated total energy expenditure (estimated from calculated BMR and self-reported physical activity level) ${ }^{(26)}$.

\section{Determination of the end point}

Information on incident cases of coronary events occurring within the cohort until the end of follow-up (31 December 2013) was obtained from national registries - that is, the Swedish Hospital Discharge Register and the Cause of Death Register. A coronary event was defined as a fatal or non-fatal MI (International Classification of Diseases (ICD)-9 codes: 410A-410X; ICD-10: I21) or death attributable to ischaemic heart disease (ICD-9 codes: 410-414; ICD-10: I20-I25).

\section{Statistical analysis}

Differences in the baseline characteristics between the coronary event cases and the non-cases and across intake groups of sucrose were examined using an ANOVA for continuous variables and $\mathrm{a} \chi^{2}$ analysis for categorical variables. The skewed food variables were logarithmically transformed to normalise the distribution. A Cox's proportional hazards regression was used to model the association between sucrose, sucrosecontaining food sources and coronary event risk. The proportional hazards assumption was assessed using Schoenfeld statistics. To fulfil the proportional hazards assumption, the model was stratified for sex and smoking. The HR and 95\% CI were calculated using the lowest categories as the reference group, and the follow-up time was used as the time scale. Trend was calculated using the median values assigned to each sucrose category and modelled as a continuous variable. In the basic model, we adjusted for total energy intake (continuous), sex, screening age (continuous), dietary data collection method (before or after 1 September 1994) and data collection season (spring/summer/fall/winter). For the final model, a multivariable analysis was conducted to adjust for other potential confounders (i.e. smoking (categorical), alcohol consumption (categorical), leisure-time physical activity (categorical) and educational level (categorical)) selected from the literature for CVD risk factors. We additionally adjusted for dietary factors associated with CVD, including wholegrains, fruits and vegetables, coffee, fermented milk, fish and meat ${ }^{(18,27,28)}$. We also included waist circumference (continuous) in a separate model because this parameter could be a confounder or be within the causal pathway for incident coronary events. In the sensitivity analyses, we excluded potential energy misreporters (for definition, see above) and individuals who reported a substantial dietary change in the past. This excluded group accounted for $35 \%$ of the study sample.

A restricted cubic spline was computed to examine the shape of the association between sucrose intake and coronary event risk, controlling for the confounders similar to those in the complete multivariable analysis; four knots were fitted on the basis of Harrell's recommended percentiles ${ }^{(29)}$, using the median intake $8.2 \mathrm{E} \%$ as the reference point. On the basis of the non-linear shape of the association, we also used the group with medium sucrose intake (7.5-10 E\%) as the reference group in the analyses with categorical sucrose intakes.

To examine whether the associations were specific to certain subgroups of the population, we performed stratified analyses to assess the potential effect modifications using predefined covariates. These effect modifiers were selected as relevant lifestyle risk factors for coronary events and included leisuretime physical activity, BMI categories, educational level, alcohol habits, smoking habits, intake of fat (E\%; tertiles), and intakes of fruits and vegetables ( $\mathrm{g} / \mathrm{MJ}$; tertiles). Interaction tests between sucrose intake and these lifestyle factors on coronary event risk were conducted by introducing a multiplicative factor in the model with the continuous variables of sucrose and lifestyle factors. All the analyses were conducted using SPSS Statistics (version 22; IBM Corporation) and Stata/SE (version 12.1; StatCorp LP). All the tests were two-sided, and $P$-values $<0 \cdot 05$ were considered statistically significant.

\section{Results}

\section{Baseline characteristics}

The mean age of the study population at baseline was 58 (SD 7.6) years (range 44.3-73.6), with 9947 (38\%) males and 16243 (62\%) females. During an average of 17 years of follow-up (range 0-22.8 years and 457131 person-years), we ascertained 2493 incident cases of coronary events (1504 men and 989 women). The baseline characteristics are summarised in Table 1 according to the amount of sucrose intake. Compared with individuals with lower sucrose consumption, individuals with higher sucrose consumption were more often smokers, had lower educational levels and were less likely to be alcohol consumers. Among men, higher sucrose intake was associated with lower waist circumference; this association was not affected after excluding diet changers and energy misreporters. Sucrose intake was inversely associated with wholegrains, meat and fish intakes. However, the highest median intake of fruits and vegetables and fermented milk and the lowest frequency of 
Table 1. Baseline characteristics of men and women in the Malmö and Diet Cancer cohort according to sucrose intake levels

(Mean values and standard deviations; medians and interquartile range (IQR); numbers and percentages)

\begin{tabular}{|c|c|c|c|c|c|c|c|c|c|c|c|c|c|c|}
\hline & \multicolumn{7}{|c|}{ Men } & \multicolumn{7}{|c|}{ Women } \\
\hline & \multicolumn{2}{|c|}{$\begin{array}{c}<5 \mathrm{E} \% \\
(n 1826 ; 18.4 \%)\end{array}$} & \multicolumn{2}{|c|}{$\begin{array}{c}7.5-10 \text { E\% } \\
(n 2580 ; 25.9 \%)\end{array}$} & \multicolumn{2}{|c|}{$\begin{array}{c}>15 \mathrm{E} \% \\
\text { (n } 465 ; 4.7 \% \text { ) }\end{array}$} & \multirow[b]{2}{*}{$P^{*}$} & \multicolumn{2}{|c|}{$\begin{array}{c}<5 \mathrm{E} \% \\
(n 1458 ; 9.0 \%)\end{array}$} & \multicolumn{2}{|c|}{$\begin{array}{c}7.5-10 \text { E\% } \\
(n 5137 ; 31.6 \%)\end{array}$} & \multicolumn{2}{|c|}{$\begin{array}{c}>15 \mathrm{E} \% \\
(n 834 ; 5 \cdot 1 \%)\end{array}$} & \multirow[b]{2}{*}{$P^{*}$} \\
\hline & Mean & SD & Mean & SD & Mean & SD & & Mean & SD & Mean & SD & Mean & SD & \\
\hline Sucrose (E\%) & 3.8 & 0.9 & 8.7 & 0.7 & $18 \cdot 0$ & $3 \cdot 2$ & $<0.01$ & 4.0 & 0.8 & 8.7 & 0.7 & $17 \cdot 8$ & $2 \cdot 8$ & $<0.01$ \\
\hline Age (years) & 57.9 & $6 \cdot 7$ & $59 \cdot 2$ & $7 \cdot 2$ & $58 \cdot 7$ & $7 \cdot 1$ & $<0.01$ & $56 \cdot 0$ & 7.5 & $57 \cdot 6$ & 7.9 & $56 \cdot 6$ & 7.8 & $<0.01$ \\
\hline Waist $(\mathrm{cm})$ & $94 \cdot 2$ & $10 \cdot 4$ & $93 \cdot 1$ & $9 \cdot 6$ & $92 \cdot 3$ & 9.9 & $<0.01$ & $78 \cdot 1$ & $10 \cdot 7$ & 77.5 & $10 \cdot 2$ & $77 \cdot 4$ & $11 \cdot 1$ & 0.4 \\
\hline \multirow[t]{2}{*}{ BMI $\left(\mathrm{kg} / \mathrm{m}^{2}\right)$} & $26 \cdot 4$ & 3.6 & $26 \cdot 0$ & 3.3 & 25.8 & 3.5 & $<0.01$ & $25 \cdot 5$ & $4 \cdot 4$ & $25 \cdot 3$ & $4 \cdot 1$ & $25 \cdot 0$ & 4.6 & 0.02 \\
\hline & Median & IQR & Median & IQR & Median & IQR & & Median & IQR & Median & IQR & Median & IQR & \\
\hline Fruits/vegetables $(\mathrm{g} / \mathrm{d})$ & 279 & $191 ; 388$ & 329 & $232 ; 457$ & 289 & $195 ; 417$ & $<0.01$ & 300 & 207; 409 & 384 & $284 ; 509$ & 327 & 223; 441 & $<0.01$ \\
\hline Wholegrains (portions/d) & 0.68 & $0.17 ; 1.63$ & 0.74 & $0.23 ; 1.60$ & 0.40 & $0.06 ; 1.08$ & $<0.01$ & 0.72 & $0.28 ; 1.39$ & 0.75 & $0.32 ; 1.35$ & 0.48 & $0.11 ; 0.96$ & $<0.01$ \\
\hline Coffee $(g / d)$ & 500 & $300 ; 800$ & 450 & $250 ; 675$ & 500 & $300 ; 900$ & $<0.01$ & 450 & $263 ; 750$ & 450 & $257 ; 675$ & 450 & $250 ; 704$ & $<0.01$ \\
\hline Fermented milk (g/d) & 0.0 & $0.0 ; 107$ & $35 \cdot 7$ & $0.0 ; 143$ & 0.0 & $0.0 ; 137$ & $<0.01$ & 36.6 & $0.0 ; 125$ & 71.4 & $0.0 ; 150$ & 50 & $0.0 ; 146$ & $<0.01$ \\
\hline Meat $(\mathrm{g} / \mathrm{d})$ & 168 & $124 ; 219$ & 152 & $116 ; 195$ & 143 & $105 ; 188$ & $<0.01$ & 115 & $83 \cdot 8 ; 152$ & 107 & $80 \cdot 3 ; 137$ & 103 & $77 \cdot 1 ; 131$ & $<0.01$ \\
\hline Fish $(g / d)$ & $39 \cdot 6$ & $18 \cdot 8 ; 68.0$ & $38 \cdot 2$ & $18 \cdot 6 ; 63 \cdot 2$ & 31.9 & $12 \cdot 0 ; 56 \cdot 8$ & $<0.01$ & 32.5 & $15 \cdot 7 ; 54.0$ & 34.0 & $18.8 ; 54.4$ & $27 \cdot 4$ & $9.5 ; 45.0$ & $<0.01$ \\
\hline Sweets $(\mathrm{g} / \mathrm{d})$ & 0.0 & $0.0 ; 2.4$ & 1.7 & $0.0 ; 6.7$ & 3.3 & $0.0 ; 12.9$ & $<0.01$ & 0.0 & $0.0 ; 2.7$ & $2 \cdot 7$ & $0.0 ; 7.7$ & 8.0 & $1.7 ; 31.5$ & $<0.01$ \\
\hline Chocolates (g/d) & 1.7 & $0 \cdot 0 ; 4 \cdot 8$ & $5 \cdot 4$ & $1 \cdot 7 ; 12 \cdot 0$ & $6 \cdot 0$ & $1 \cdot 4 ; 18 \cdot 3$ & $<0.01$ & 1.3 & $0.0 ; 3.5$ & $5 \cdot 2$ & $1 \cdot 9 ; 10 \cdot 8$ & $7 \cdot 3$ & $2 \cdot 3 ; 18.9$ & $<0.01$ \\
\hline Sugar and jam (g/d) & $10 \cdot 5$ & $2 \cdot 4 ; 21 \cdot 6$ & 33.6 & $18.9 ; 49.5$ & 63.5 & $37 \cdot 6 ; 90 \cdot 3$ & $<0.01$ & $6 \cdot 6$ & $1 \cdot 3 ; 14 \cdot 2$ & $18 \cdot 9$ & $9 \cdot 3 ; 30 \cdot 3$ & 38.6 & $19 \cdot 6 ; 62 \cdot 7$ & $<0.01$ \\
\hline Fruit juice $(\mathrm{g} / \mathrm{d})$ & 0.0 & $0.0 ; 21.4$ & 0.4 & $0.0 ; 85.7$ & 0.0 & $0.0 ; 78.6$ & $<0.01$ & 0.0 & $0.0 ; 28.6$ & $28 \cdot 6$ & $0.0 ; 121$ & 0.66 & $0.0 ; 122$ & $<0.01$ \\
\hline Sugar-sweetened beverages $(\mathrm{g} / \mathrm{d})$ & 0.0 & $0.0 ; 7 \cdot 2$ & 42.9 & $0 \cdot 0 ; 141$ & 250 & $57 \cdot 1 ; 532$ & $<0.01$ & 0.0 & $0.0 ; 0.0$ & $14 \cdot 3$ & $0 \cdot 0 ; 75 \cdot 7$ & 188 & $42 \cdot 9 ; 386$ & $<0.01$ \\
\hline \multirow[t]{2}{*}{ Cakes and pastries $(\mathrm{g} / \mathrm{d})$} & $15 \cdot 5$ & $5 \cdot 0 ; 31.9$ & $37 \cdot 6$ & $20 \cdot 1 ; 59 \cdot 8$ & 37.4 & $15 \cdot 0 ; 71 \cdot 0$ & $<0.01$ & $11 \cdot 8$ & $4.4 ; 22 \cdot 9$ & $34 \cdot 4$ & $19 \cdot 5 ; 53 \cdot 1$ & $35 \cdot 6$ & $18 \cdot 1 ; 61 \cdot 9$ & $<0.01$ \\
\hline & $n$ & $\%$ & $n$ & $\%$ & $n$ & $\%$ & & $n$ & $\%$ & $n$ & $\%$ & $n$ & $\%$ & \\
\hline $\begin{array}{l}\text { Smokers } \\
\text { Educational level }\end{array}$ & 590 & $32 \cdot 3$ & 661 & $25 \cdot 6$ & 212 & $45 \cdot 6$ & $<0.01$ & 546 & 37.4 & 1271 & $24 \cdot 7$ & 351 & $42 \cdot 1$ & $\begin{array}{l}<0.01 \\
<0.01\end{array}$ \\
\hline $\begin{array}{l}\text { Educational level } \\
\text { Elementary or less }\end{array}$ & 786 & $43 \cdot 0$ & 1087 & $42 \cdot 1$ & 260 & 55.9 & & 549 & 37.7 & 1944 & $37 \cdot 8$ & 378 & $45 \cdot 3$ & \\
\hline University degree & 256 & $14 \cdot 0$ & 361 & $14 \cdot 0$ & 44 & 9.5 & & 285 & $19 \cdot 5$ & 740 & $14 \cdot 4$ & 89 & $10 \cdot 7$ & \\
\hline Alcohol habits & & & & & & & $<0.01$ & & & & & & & $<0.01$ \\
\hline Zero consumers & 64 & 3.5 & 101 & 3.9 & 52 & $11 \cdot 2$ & & 98 & 6.7 & 336 & 6.5 & 105 & $12 \cdot 6$ & \\
\hline Highest quintile & 517 & $28 \cdot 3$ & 450 & $17 \cdot 4$ & 56 & $12 \cdot 0$ & & 426 & $29 \cdot 2$ & 893 & $17 \cdot 4$ & 92 & 11.0 & \\
\hline Leisure-time physical activity & & & & & & & $<0.01$ & & & & & & & $<0.01$ \\
\hline Lowest quintile & 456 & $25 \cdot 0$ & 443 & $17 \cdot 2$ & 121 & $26 \cdot 0$ & & 364 & $25 \cdot 0$ & 930 & $18 \cdot 1$ & 219 & $26 \cdot 3$ & \\
\hline Highest quintile & 293 & $16 \cdot 0$ & 525 & $20 \cdot 3$ & 86 & 18.5 & & 266 & $18 \cdot 2$ & 1066 & $20 \cdot 8$ & 165 & $19 \cdot 8$ & \\
\hline
\end{tabular}

$\mathrm{E} \%$, energy percentage.

${ }^{*} P$ values were calculated using $x^{2}$ tests for categorical variables and ANOVA for continuous variables. 
individuals with low leisure-time physical activity were observed among individuals with moderate sucrose intake. Higher sucrose intake was significantly and positively associated with the consumption of all sucrose-containing food sources - that is, sweets, chocolates, sugar and jam, fruit juice, sugar-sweetened beverages, and cakes and pastries (Table 1).

\section{Sucrose and the risk of incident coronary events}

The incidence rates of coronary events within the lowest and highest intakes of sucrose $\mathrm{E} \%$ were $6 \cdot 1 v .7 \cdot 5$ cases/1000 person-years, respectively. In the minimally adjusted model, an intake $>15 \mathrm{E} \%$ compared with $<5 \mathrm{E} \%$ was associated with a $48 \%$ increased coronary event risk $\left(P_{\text {trend }}<0.001\right)$. In addition, a tendency towards a decreased risk of coronary events in the groups consuming 5-7.5 E\% and 7.5-10 E\% compared with the lowest category was observed (Table 2). After adjusting for potential confounders (including waist circumference), the association was attenuated; however, the risk in the highest group ( $>15 \mathrm{E} \%)$ compared with the lowest intake group remained significant (HR 1.37; $95 \%$ CI 1.13, 1.66). Among the potential confounders, smoking, waist circumference, alcohol consumption, leisure-time physical activity and educational level affected the HR. The risks of coronary events across the other groups ( $<15 \mathrm{E} \%)$ were similar; thus, an elevated risk was observed in the group with intakes $>15 \mathrm{E} \%$ (Table 2). The associations were attenuated between the extreme intake groups when the energy misreporters and participants with a reported diet change in the past (35\% of the study sample) were excluded from the analysis (HR $1.25 ; 95 \%$ CI $0.98,1.61$ ). However, when using the middle group (7.5-10 E\%) as the reference, the risk estimate for the highest intake group was similar after excluding diet changers and energy misreporters (HR 1.30; $95 \%$ CI 1.04, 1.63) (Table 2).

Fig. 1 shows the non-linear relationship between sucrose intake and the risk of coronary events fitting a four-knot cubic spline with the reference at $8.2 \mathrm{E} \%$ (median intake). This analysis indicated that the increasing coronary event risk associated with sucrose intake increased above the median intake, with statistically significant levels above $13 \mathrm{E} \%$.

The sucrose-containing foods or beverages were not significantly associated with coronary event risk after adjusting for potential confounders. However, we observed a trend towards increased risk of coronary events and the consumption of sweets (HR 1.17; 95\% CI 0.99, 1.39; $\left.\quad P_{\text {trend }}=0.07\right)$, sugar-sweetened beverages (HR 1.12; $95 \%$ CI 0.97, 1.28; $P_{\text {trend }}=0 \cdot 06$ ), and sugar and jam (HR 1.12;95\% CI 0.96, 1.32; $\left.P_{\text {trend }}=0.50\right)$ for the highest $v$. lowest intake groups (Table 3 ).

\section{Population sub-group analysis}

There were no statistically significant interactions (using continuous variables) between the potential effect modifiers - that is, education, leisure-time physical activity, BMI, smoking, fruit and vegetable intakes, fat intake and sucrose intake on coronary event risk (online Supplementary Table S1). Although no statistically significant interaction was observed between sucrose intake and educational level $(P=0 \cdot 08)$, stronger

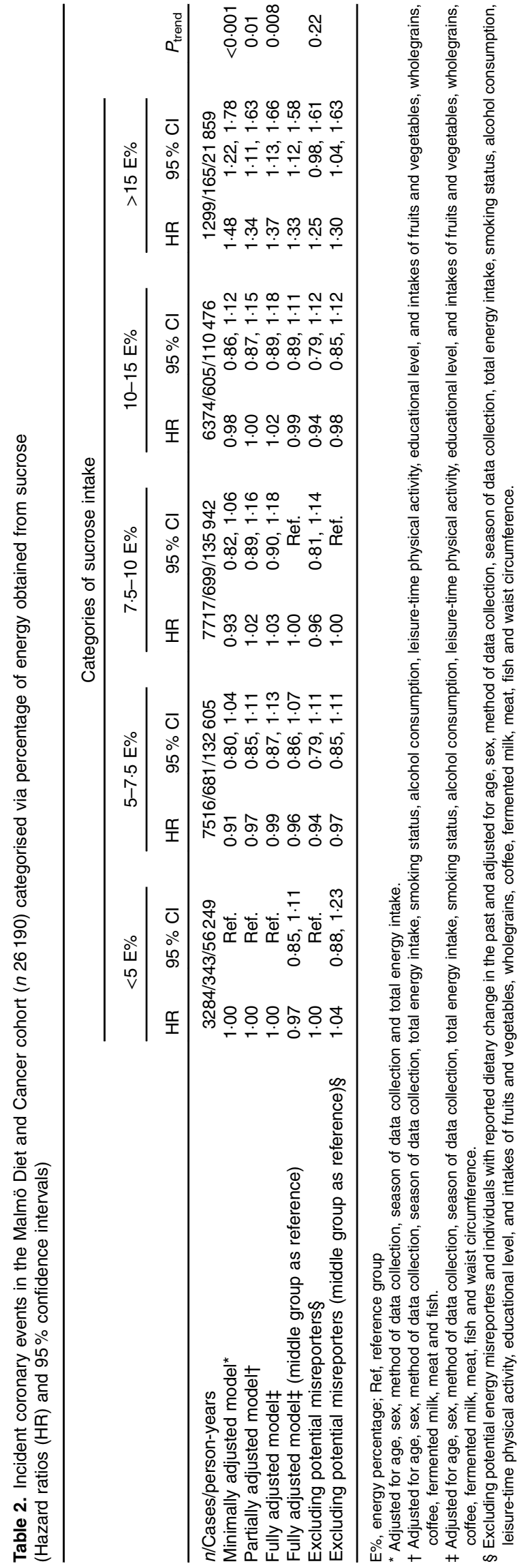




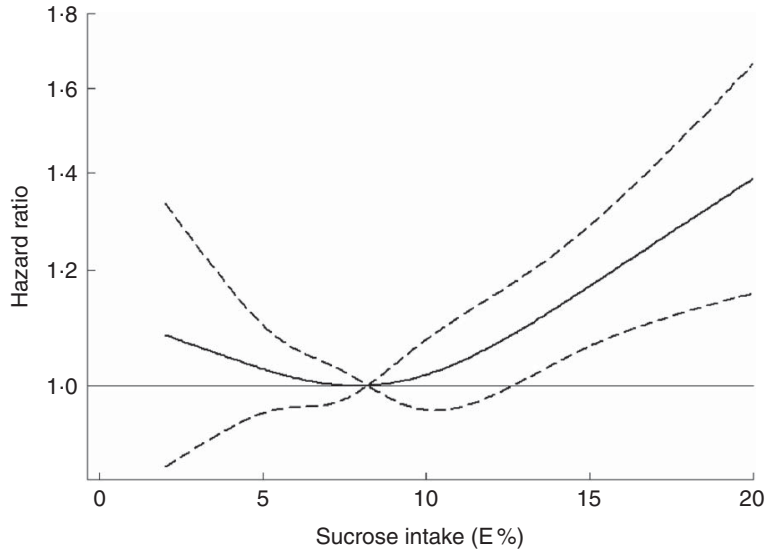

Fig. 1. Association (hazard ratio (-) and $95 \%$ confidence interval (---)) between sucrose intake and risk of coronary events modelled using a restricted cubic spline with four knots and using the median intake ( $8.2 \%$ energy; $\mathrm{E} \%$ ) as the reference point.

associations were suggested for individuals with a high educational level (HR for highest $v$. lowest intake $=2 \cdot 19$; $95 \% \mathrm{CI}$ 1.10, 4.40). Among individuals with high levels of physical activity, a significantly increased risk was observed at a moderate sucrose intake (5-7.5 E\%), with a $38 \%$ (HR 1.38; $95 \%$ CI $1 \cdot 00,1.91)$ increased risk compared with individuals with $<5 \mathrm{E}$ $\%$. However, a moderate amount of sucrose (5-10 E\%) was not associated with increased coronary event risk compared with low sucrose intake $(<5 \mathrm{E} \%)$ in any other strata. Overall, the results indicated that the association between sucrose intake and coronary event risk is independent of lifestyle factors, and none of the potential effect modifiers strengthened or weakened the association between sucrose and coronary events.

\section{Discussion}

In this prospective study, high sucrose intake was associated with an increased risk of incident coronary events. In all, $5 \%$ of the population with an intake $>15 \mathrm{E} \%$ had a $37 \%$ increased risk compared with individuals with an intake $<5 \mathrm{E} \%$. However, the non-linear association indicated no benefit of having a lower sucrose intake than the median intake $(8.2 \mathrm{E} \%)$. None of the sucrose-containing food sources or beverages were significantly associated with coronary event risk; therefore, no single food source could explain the increased risk observed for sucrose.

A few studies have investigated the association between the consumption of sugars (including sucrose), sugar-sweetened beverages and sugar-rich foods and CVD risk. A systematic literature review and meta-analysis of four prospective studies revealed a $17 \%$ increased risk of $\mathrm{CHD}$ for every serving per day of sugar-sweetened beverages ${ }^{(30,31)}$. None of the included studies were, however, conducted among European populations. Studies examining specific sugars are even rarer. Liu et al. ${ }^{(32)}$ did not observe an association between sucrose and CVD risk among 75521 women from the Nurses' Health Study. In Sweden, sugar-sweetened beverages are sweetened by sucrose. We observed a stronger association for sucrose intake than sugar-sweetened beverage intake, which might indicate that the combined sucrose intake might be more important than only sugar-sweetened beverages. However, sugar-sweetened beverage intake was reported in the $7-\mathrm{d}$ food diary, and misclassifications might occur, especially among individuals with low intake.

Added sugar in the form of sucrose is the main source of fructose in the Swedish diet ${ }^{(33)}$. The evidence for the adverse effects of fructose is mainly based on animal studies. It is unclear whether the same effects, and to a similar extent, occur in humans, especially in the long-term ${ }^{(34-36)}$. The conclusion from short-term clinical trials so far has been that fructose has no additional adverse health effects on blood pressure or body weight when exchanged isoenergetically for other carbohydrates. However, significant effects of fructose have been observed in hyperenergetic trials ${ }^{(37,38)}$. In addition, very high fructose intakes $(>100 \mathrm{~g} / \mathrm{d})$ seem to increase LDL-cholesterol when isoenergetically replaced with other carbohydrates ${ }^{(39)}$. A systematic review concluded that dietary sugar raised blood lipids - a relationship that seems to be independent of the effect of sugars on body weight ${ }^{(40)}$. Within the MDC cohort, high sucrose intake was associated with higher TAG and lower HDLcholesterol concentrations ${ }^{(18)}$. In animal studies, high sucrose intake was associated with the development of hypertension, which is a risk factor for $\mathrm{CVD}^{(41)}$. An epidemiological study also suggested that a high intake of sugar and sugar-sweetened beverages was associated with an increase in blood pressure ${ }^{(31,42)}$. In contrast, Forman et al. ${ }^{(43)}$ observed no link between fructose and development of hypertension. Another possible mechanism is the influence of sugar on inflammation. A randomised-controlled trial conducted on healthy young men ${ }^{(44)}$ and a prospective study conducted on men ${ }^{(16)}$ reported a higher degree of inflammation after the consumption of sugarsweetened beverages. The sugar-sweetened beverages induced hyperglycaemia and in turn influenced inflammation, as demonstrated in a randomised trial conducted on healthy women $^{(45)}$. Inflammation is a major factor in the pathogenesis of CHD and can be used as a marker for the increased risk of coronary events $^{(46)}$. However, a study conducted within the MDC cohort showed no difference in the level of inflammatory markers between those with $>$ and $<10 \mathrm{E} \%$ of sucrose intake ${ }^{(47)}$. This absence of association may reflect the small sample size of the study; furthermore, the age range of the study participants (63-68 years) differed from the age range (44-73 years) in the present study.

The present study has several strengths. First, as a prospective cohort study, the likelihood of reverse causality was minimised because information on the exposure and risk factors was collected at baseline before the development of a coronary event. Second, the large sample size of the cohort and the number of incident coronary event cases provided a large statistical power to detect the associated effects of the sugar exposure. Third, cases of incident coronary events were obtained from national hospital registers. The coronary event statuses have been validated to have a high sensitivity and a high predictive value, thereby reducing the misclassification of the disease status ${ }^{(48,49)}$. Fourth, the MDC data set contained information on potential confounders (age, season, diet method, energy intake, smoking, alcohol consumption, leisure-time 
Table 3. Incident coronary events in the Malmö Diet and Cancer cohort ( $n$ 26 190) categorised by intake of sucrose food sources (Hazard ratios (HR) and $95 \%$ confidence intervals)

\begin{tabular}{|c|c|c|c|c|c|c|c|c|c|}
\hline & \multicolumn{8}{|c|}{ Categories of intake } & \multirow[b]{3}{*}{$P_{\text {trend }}$} \\
\hline & \multicolumn{2}{|c|}{1} & \multicolumn{2}{|c|}{2} & \multicolumn{2}{|c|}{3} & \multicolumn{2}{|c|}{4} & \\
\hline & $\mathrm{HR}$ & $95 \% \mathrm{Cl}$ & $\mathrm{HR}$ & $95 \% \mathrm{Cl}$ & $\mathrm{HR}$ & $95 \% \mathrm{Cl}$ & HR & $95 \% \mathrm{Cl}$ & \\
\hline \multicolumn{10}{|l|}{ Sweets } \\
\hline Intakes $(\mathrm{g} / \mathrm{d})^{\star}$ & \multirow{2}{*}{\multicolumn{2}{|c|}{$\begin{array}{c}\text { Zero consumers } \\
8627 / 958 / 147846\end{array}$}} & \multirow{2}{*}{\multicolumn{2}{|c|}{$\begin{array}{c}0.1-5 \mathrm{~g} \\
8543 / 802 / 150991\end{array}$}} & \multirow{2}{*}{\multicolumn{2}{|c|}{$\begin{array}{c}5-20 \mathrm{~g} \\
7095 / 567 / 124906\end{array}$}} & \multirow{2}{*}{\multicolumn{2}{|c|}{$\begin{array}{c}>20 \mathrm{~g} \\
1925 / 166 / 33388\end{array}$}} & \\
\hline$n /$ Cases/person-years & & & & & & & & & \\
\hline Minimally adjusted $\dagger$ & 1.00 & Ref. & 0.91 & $0.83,1.00$ & 0.99 & $0.90,1.11$ & $1 \cdot 30$ & $1 \cdot 10,1.54$ & 0.001 \\
\hline Fully adjusted $\ddagger$ & 1.00 & Ref. & 0.94 & $0.86,1.04$ & 0.96 & $0.87,1.07$ & $1 \cdot 17$ & $0.99,1.39$ & 0.07 \\
\hline \multicolumn{10}{|l|}{ Chocolates } \\
\hline Intakes $(\mathrm{g} / \mathrm{d})^{*}$ & \multirow{2}{*}{\multicolumn{2}{|c|}{$\begin{array}{l}\text { Zero consumers } \\
4583 / 515 / 77149\end{array}$}} & \multirow{2}{*}{\multicolumn{2}{|c|}{$\begin{array}{c}0 \cdot 1-5 \mathrm{~g} \\
8046 / 768 / 140795\end{array}$}} & \multirow{2}{*}{\multicolumn{2}{|c|}{$\begin{array}{c}5-20 \mathrm{~g} \\
11196 / 977 / 197592\end{array}$}} & \multirow{2}{*}{\multicolumn{2}{|c|}{$\begin{array}{c}>20 \mathrm{~g} \\
2365 / 233 / 41595\end{array}$}} & \\
\hline$n /$ Cases/person-years & & & & & & & & & \\
\hline Minimally adjusted $\dagger$ & 1.00 & Ref. & 0.78 & $0.69,0.87$ & 0.85 & $0.76,0.94$ & 0.93 & $0.79,1.09$ & 0.58 \\
\hline Fully adjusted $\ddagger$ & 1.00 & Ref. & 0.84 & $0.75,0.94$ & 0.91 & $0.82,1.01$ & 0.96 & $0.82,1.13$ & 0.55 \\
\hline \multicolumn{10}{|l|}{ Sugar and jam } \\
\hline Intakes $(\mathrm{g} / \mathrm{d})^{*}$ & \multirow{2}{*}{\multicolumn{2}{|c|}{$\begin{array}{c}0-10 \mathrm{~g} \\
5458 / 467 / 95942\end{array}$}} & \multirow{2}{*}{\multicolumn{2}{|c|}{$\begin{array}{c}10-20 \mathrm{~g} \\
5981 / 538 / 106028\end{array}$}} & \multirow{2}{*}{\multicolumn{2}{|c|}{$\begin{array}{c}20-60 \mathrm{~g} \\
12864 / 1242 / 223217\end{array}$}} & \multirow{2}{*}{\multicolumn{2}{|c|}{$\begin{array}{c}>60 \mathrm{~g} \\
1887 / 246 / 31944\end{array}$}} & \\
\hline$n /$ Cases/person-years & & & & & & & & & \\
\hline Minimally adjusted $\dagger$ & 1.00 & Ref. & $1 \cdot 11$ & $0.97,1.26$ & 1.03 & $0.92,1.15$ & $1 \cdot 19$ & $1.02,1.39$ & 0.11 \\
\hline Fully adjusted $\ddagger$ & 1.00 & Ref. & $1 \cdot 14$ & $1.00,1.30$ & 1.02 & $0.91,1.15$ & $1 \cdot 12$ & $0.96,1.32$ & 0.50 \\
\hline \multicolumn{10}{|l|}{ Fruit juice } \\
\hline Intakes $(\mathrm{g} / \mathrm{d})^{*}$ & \multirow{2}{*}{\multicolumn{2}{|c|}{$\begin{array}{c}\text { Zero consumers } \\
11700 / 1290 / 200917\end{array}$}} & \multirow{2}{*}{\multicolumn{2}{|c|}{$\begin{array}{c}0.1-50 \mathrm{~g} \\
5647 / 501 / 100294\end{array}$}} & \multirow{2}{*}{\multicolumn{2}{|c|}{$\begin{array}{c}50-250 \mathrm{~g} \\
7556 / 601 / 133574\end{array}$}} & \multirow{2}{*}{\multicolumn{2}{|c|}{$\begin{array}{c}>250 \mathrm{~g} \\
1287 / 101 / 22345\end{array}$}} & \\
\hline$n /$ Cases/person-years & & & & & & & & & \\
\hline Minimally adjusted $\dagger$ & 1.00 & Ref. & 0.86 & $0.78,0.96$ & 0.84 & $0.76,0.93$ & 0.88 & $0.72,1.07$ & 0.02 \\
\hline Fully adjusted $\ddagger$ & 1.00 & Ref. & 0.95 & $0.85,1.05$ & 0.94 & $0.85,1.04$ & 0.92 & $0.75,1.12$ & 0.25 \\
\hline \multicolumn{10}{|c|}{ Sugar-sweetened beverages } \\
\hline Intakes $(\mathrm{g} / \mathrm{d})^{\star}$ & \multirow{2}{*}{\multicolumn{2}{|c|}{$\begin{array}{c}\text { Zero consumers } \\
11849 / 1127 / 205934\end{array}$}} & & & & & & & \\
\hline$n /$ Cases/person-years & & & & 78086 & & 35793 & & 37317 & \\
\hline Minimally adjusted $\dagger$ & 1.00 & Ref. & 0.91 & $0.81,1.02$ & 1.04 & $0.94,1.14$ & $1 \cdot 19$ & $1.04,1.36$ & 0.003 \\
\hline Fully adjusted $\neq$ & 1.00 & Ref. & 0.94 & $0.84,1.06$ & 1.04 & $0.95,1.15$ & $1 \cdot 12$ & $0.97,1.28$ & 0.06 \\
\hline Cakes and pastries & & & & & & & & & \\
\hline Intakes $(\mathrm{g} / \mathrm{d})^{*}$ & & & & & & & & & \\
\hline$n /$ Cases/person-years & & 2135 & & 49625 & & 40214 & & 35157 & \\
\hline Minimally adjusted $\dagger$ & 1.00 & Ref. & 0.92 & $0.83,1.02$ & 0.89 & $0.80,0.99$ & 0.87 & $0.75,1.02$ & 0.03 \\
\hline Fully adjusted $\neq$ & 1.00 & Ref. & 0.97 & $0.87,1.08$ & 0.96 & $0.86,1.07$ & 0.92 & $0.79,1.08$ & 0.31 \\
\hline
\end{tabular}


physical activity, education, waist circumference and other dietary factors) that we adjusted for. The data set also contained variables that facilitated the sensitivity analyses, for example, whether the participants had changed their food habits in the past and energy misreporting. However, the results did not change markedly when these individuals were excluded, especially not when using the middle group as a reference group. Moreover, the use of a validated modified diet history method $^{(22)}$ provided a detailed exposure assessment of sucrosecontaining foods and beverages. In addition, the food database created for the MDC cohort was comprehensive, and any missing foods were updated into the database. For all the foods included in the database, sucrose was measured directly, calculated from recipes or obtained from the table of contents.

There are also certain limitations to the present study. Dietary intake was self-reported; therefore, we cannot rule out measurement errors. There was a tendency for women and obese individuals to under-report the consumption of added sugars and snacks consumed between meals ${ }^{(50)}$. Measurement errors due to self-reporting could lead to the misclassification of sucrose intake. However, this error is likely random (nondifferential) and is not associated with the outcome status, because the coronary events were recorded after baseline data were collected, and individuals with prevalent coronary events were excluded. This non-differential error could have attenuated the association observed in the present study; therefore, the associated effect of high sucrose intake may be even higher than that observed in the present study. In addition, because sucrose has been used as a marker of added sugar, the consumption of added sugar was underestimated and probably the association between added sugar intake and coronary event risk as well. To obtain more reliable estimates on sucrose intake, biomarkers, for example, the urinary excretion of sucrose, could be used as a surrogate measure of sucrose intake $^{(51)}$. Dietary intake was only assessed during the baseline examinations and may have changed during the follow-up period. For example, the dietary habits in Sweden during which the baseline data were collected (1991-1996) are different from the current dietary habits - for instance, there has been an increase in the consumption of sugar-sweetened beverages from 50 litres/person per year in 1990 to 87 litres/person per year in $2013^{(52)}$. Although we had detailed information and controlled for the most important confounders, we cannot rule out the possibility of residual confounding factors. Furthermore, the results were based on a cohort of participants who were between the ages of 44 and 73 years, potentially limiting the generalisability of the results to younger and older populations. Additional studies are required in other age groups to confirm the current findings.

The median intake of sucrose reported in the present study was $8.2 \mathrm{E} \%$. This finding is similar to a national survey conducted in Sweden in 1997 (median intake of sucrose was $8 \mathrm{E} \%$ for both men and women) ${ }^{(53)}$. On the basis of results from a national survey in 2010, the intake of sucrose had decreased to $7 \cdot 3 \mathrm{E} \%$. In the present study, we could not differentiate whether sucrose was obtained from naturally occurring sources (intrinsic) or from added sugar (extrinsic). However, in the national survey from 2010 , only $20 \%$ of the sucrose consumed was obtained from natural sources - that is, fruits, vegetables and juices. The mean intake of added sugar (sucrose, fructose, glucose, starch hydrolysates and other isolated sugar preparations added during food preparation) was estimated to be $8.9 \mathrm{E} \%{ }^{(33)}$. In the present study, only forty-one individuals ( $0 \cdot 16 \%$ of the total study population) had a sucrose intake $>25$ $\mathrm{E} \%$ (maximum sucrose intake of $38 \mathrm{E} \%$ ). Therefore, we could not examine the coronary event risk for individuals with a sucrose intake level $>25 \mathrm{E} \%$.

In conclusion, these data show a non-linear association between sucrose intake and coronary event incidence after controlling for potential confounders. These results further indicate that a sucrose intake above $15 \mathrm{E} \%$ increases coronary event risk. In addition, these data do not suggest that a sucrose intake between 5 and $10 \mathrm{E} \%$ increases the coronary event risk. There was no strong evidence of heterogeneity of effect for any of the lifestyle factors. However, further long-term studies are required to determine whether other factors, for example, genetic factors, influence the susceptibility to coronary events, even with moderate sugar consumption. Further investigation is also warranted to determine the effects of sucrose from different sources on the incidence of coronary events.

\section{Acknowledgements}

This study was funded by the Swedish Research Council (E. S., grant number K2012-99X-220018-01-3), the Swedish Society for Medical Research (E. S.), the Albert Påhlsson Foundation (E. S.), Crafoord Foundation (E. S.) and ALF government grants (E. S.). The funding sources had no involvement in the study design, the collection, analyses and interpretation of the data, writing the report or the decision to submit the article for publication.

K. W. and E. S. formulated the research question and designed the study; K. W. analysed the data and wrote the first draft of the article; I. D. provided help with the statistical analyses; E. S., I. D. and G. E. provided critical review. All the authors read and approved the final version of the manuscript. E. S. has the primary responsibility for the final content.

The authors declare that there are no conflicts of interest.

\section{Supplementary material}

For supplementary material/s referred to in this article, please visit http://dx.doi.org/doi:10.1017/S0007114516003561

\section{References}

1. World Health Organization (2013) Cardiovascular disease. http://www.who.int/cardiovascular_diseases/en/ (accessed January 2015).

2. Johnson RJ, Segal MS, Sautin Y, et al. (2007) Potential role of sugar (fructose) in the epidemic of hypertension, obesity and the metabolic syndrome, diabetes, kidney disease, and cardiovascular disease. Am J Clin Nutr 86, 899-906.

3. Howard BV \& Wylie-Rosett J (2002) Sugar and cardiovascular disease: a statement for healthcare professionals from the Committee on Nutrition of the Council on Nutrition, Physical Activity, and Metabolism of the American Heart Association. Circulation 106, 523-527. 
4. Gibson SA (2007) Dietary sugars intake and micronutrient adequacy: a systematic review of the evidence. Nutr Res Rev 20, 121-131.

5. Louie JC \& Tapsell LC (2015) Association between intake of total vs added sugar on diet quality: a systematic review. Nutr Rev 73, 837-857.

6. Rennie KL \& Livingstone MB (2007) Associations between dietary added sugar intake and micronutrient intake: a systematic review. Br J Nutr 97, 832-841.

7. Te Morenga L, Mallard S \& Mann J (2013) Dietary sugars and body weight: systematic review and meta-analyses of randomised controlled trials and cohort studies. BMJ 346, e7492.

8. Yang Q, Zhang Z, Gregg EW, et al. (2014) Added sugar intake and cardiovascular diseases mortality among US adults. JAMA Intern Med 174, 516-524.

9. World Health Organization (2015) Guideline: Sugar Intake for Adults and Children. Geneva: WHO.

10. Nishida C, Uauy R, Kumanyika S, et al. (2004) The joint WHO/ FAO expert consultation on diet, nutrition and the prevention of chronic diseases: process, product and policy implications. Public Health Nutr 7, 245-250.

11. Nordic Council of Ministers (2014) Nordic Nutrition Recommendations 2012, 5th ed. Copenhagen, Denmark: Nordic Council of Ministers.

12. Sonestedt E, Overby NC, Laaksonen DE, et al. (2012) Does high sugar consumption exacerbate cardiometabolic risk factors and increase the risk of type 2 diabetes and cardiovascular disease? Food Nutr Res 56, 19104

13. Malik VS, Popkin BM, Bray GA, et al. (2010) Sugar-sweetened beverages and risk of metabolic syndrome and type 2 diabetes: a meta-analysis. Diabetes Care 33, 2477-2483.

14. Imamura F, O'Connor L, Ye Z, et al. (2015) Consumption of sugar sweetened beverages, artificially sweetened beverages, and fruit juice and incidence of type 2 diabetes: systematic review, meta-analysis, and estimation of population attributable fraction. BMJ 351, h3576.

15. Keller A, Heitmann BL \& Olsen N (2015) Sugar-sweetened beverages, vascular risk factors and events: a systematic literature review. Public Health Nutr 18, 1145-1154.

16. de Koning L, Malik VS, Kellogg MD, et al. (2012) Sweetened beverage consumption, incident coronary heart disease, and biomarkers of risk in men. Circulation 125, 1735-1741.

17. Hlebowicz J, Drake I, Gullberg B, et al. (2013) A high diet quality is associated with lower incidence of cardiovascular events in the Malmö diet and cancer cohort. PLOS ONE $\mathbf{8}$, e71095.

18. Sonestedt E, Hellstrand S, Schulz CA, et al. (2015) The association between carbohydrate-rich foods and risk of cardiovascular disease is not modified by genetic susceptibility to dyslipidemia as determined by 80 validated variants. PLOS ONE 10, e0126104.

19. Manjer J, Elmstahl S, Janzon L, et al. (2002) Invitation to a population-based cohort study: differences between subjects recruited using various strategies. Scand J Public Health 30, 103-112.

20. Manjer J, Carlsson S, Elmstahl S, et al. (2001) The Malmö Diet and Cancer Study: representativity, cancer incidence and mortality in participants and non-participants. Eur J Cancer Prev 10, 489-499.

21. Wirfalt E, Mattisson I, Johansson U, et al. (2002) A methodological report from the Malmö Diet and Cancer study: development and evaluation of altered routines in dietary data processing. Nutr J 1, 3 .

22. Callmer E, Riboli E, Saracci R, et al. (1993) Dietary assessment methods evaluated in the Malmö food study. J Intern Med 233, 53-57.
23. Riboli E, Elmstahl S, Saracci R, et al. (1997) The Malmö Food Study: validity of two dietary assessment methods for measuring nutrient intake. Int J Epidemiol 26, Suppl. 1, S161-S173.

24. Taylor HL, Jacobs DR Jr., Schucker B, et al. (1978) A questionnaire for the assessment of leisure time physical activities. J Chronic Diseases 31, 741-755.

25. Sonestedt E, Wirfalt E, Gullberg B, et al. (2005) Past food habit change is related to obesity, lifestyle and socio-economic factors in the Malmo Diet and Cancer Cohort. Public Health Nutr 8, 876-885.

26. Mattisson I, Wirfalt E, Aronsson CA, et al. (2005) Misreporting of energy: prevalence, characteristics of misreporters and influence on observed risk estimates in the Malmö Diet and Cancer Cohort. Br J Nutr 94, 832-842.

27. Mente A, de Koning L, Shannon HS, et al. (2009) A systematic review of the evidence supporting a causal link between dietary factors and coronary heart disease. Arch Intern Med 169, 659-669.

28. Sonestedt E, Wirfalt E, Wallstrom P, et al. (2011) Dairy products and its association with incidence of cardiovascular disease: the Malmö diet and cancer cohort. Eur J Epidemiol 26, 609-618.

29. Harrell FE (2001) Regression Modeling Strategies: With Applications to Linear Models, Logistic Regression, and Survival Analysis, Springer series in statistics. New York, NY: Springer.

30. Huang C, Huang J, Tian Y, et al. (2014) Sugar sweetened beverages consumption and risk of coronary heart disease: a metaanalysis of prospective studies. Atherosclerosis 234, 11-16.

31. Xi B, Huang Y, Reilly KH, et al. (2015) Sugar-sweetened beverages and risk of hypertension and CVD: a dose-response meta-analysis. Br J Nutr 113, 709-717.

32. Liu S, Willett WC, Stampfer MJ, et al. (2000) A prospective study of dietary glycemic load, carbohydrate intake, and risk of coronary heart disease in US women. Am J Clin Nutr $\mathbf{7 1}$, $1455-1461$

33. Amcoff E, Edberg A, Enghardt Barbieri H, et al. (2012) Riksmaten - vuxna 2010-11: Livsmedels- och näringsintag bland vuxna $i$ Sverige (Food and Nutrient Intake among Adults in Sweden). Uppsala, Sweden: Swedish National Food Agency.

34. Ha V, Jayalath VH, Cozma AI, et al. (2013) Fructose-containing sugars, blood pressure, and cardiometabolic risk: a critical review. Curr Hypertens Rep 15, 281-297.

35. Sun SZ \& Empie MW (2012) Fructose metabolism in humans - what isotopic tracer studies tell us. Nutr Metab (Lond) 9, 89.

36. Stanhope KL, Schwarz JM \& Havel PJ (2013) Adverse metabolic effects of dietary fructose: results from the recent epidemiological, clinical, and mechanistic studies. Curr Opin Lipidol 24, 198-206.

37. David Wang D, Sievenpiper JL, de Souza RJ, et al. (2014) Effect of fructose on postprandial triglycerides: a systematic review and meta-analysis of controlled feeding trials. Atherosclerosis 232, 125-133.

38. Sievenpiper JL, de Souza RJ, Mirrahimi A, et al. (2012) Effect of fructose on body weight in controlled feeding trials: a systematic review and meta-analysis. Ann Intern Med 156, 291-304.

39. Zhang YH, An T, Zhang RC, et al. (2013) Very high fructose intake increases serum LDL-cholesterol and total cholesterol: a meta-analysis of controlled feeding trials. I Nutr $\mathbf{1 4 3}$, $1391-1398$

40. Te Morenga LA, Howatson AJ, Jones RM, et al. (2014) Dietary sugars and cardiometabolic risk: systematic review and meta-analyses of randomized controlled trials of 
the effects on blood pressure and lipids. Am J Clin Nutr $\mathbf{1 0 0}$, 65-79.

41. Bunag RD, Tomita T \& Sasaki S (1983) Chronic sucrose ingestion induces mild hypertension and tachycardia in rats. Hypertension 5, 218-225.

42. Brown IJ, Stamler J, Van Horn L, et al. (2011) Sugar-sweetened beverage, sugar intake of individuals, and their blood pressure: international study of macro/micronutrients and blood pressure. Hypertension 57, 695-701.

43. Forman JP, Choi H \& Curhan GC (2009) Fructose and vitamin $\mathrm{C}$ intake do not influence risk for developing hypertension. J Am Soc Nephrol 20, 863-871.

44. Aeberli I, Gerber PA, Hochuli M, et al. (2011) Low to moderate sugar-sweetened beverage consumption impairs glucose and lipid metabolism and promotes inflammation in healthy young men: a randomized controlled trial. Am J Clin Nutr $\mathbf{9 4}$, 479-485.

45. Liu S, Manson JE, Buring JE, et al. (2002) Relation between a diet with a high glycemic load and plasma concentrations of high-sensitivity C-reactive protein in middle-aged women. Am J Clin Nutr 75, 492-498.

46. Kuller LH, Tracy RP, Shaten J, et al. (1996) Relation of C-reactive protein and coronary heart disease in the MRFIT nested case-control study. Multiple Risk Factor Intervention Trial. Am J Epidemiol 144, 537-547.

47. Dias JA, Wirfalt E, Drake I, et al. (2015) A high quality diet is associated with reduced systemic inflammation in middleaged individuals. Atherosclerosis 238, 38-44.

48. Hammar N, Alfredsson L, Rosen M, et al. (2001) A national record linkage to study acute myocardial infarction incidence and case fatality in Sweden. Int J Epidemiol 30, Suppl. 1, S30-S34.

49. Ludvigsson JF, Andersson E, Ekbom A, et al. (2011) External review and validation of the Swedish national inpatient register. BMC Public Health 11, 450.

50. Poppitt SD, Swann D, Black AE, et al. (1998) Assessment of selective under-reporting of food intake by both obese and non-obese women in a metabolic facility. Int $J$ Obes Relat Metab Disord 22, 303-311.

51. Tasevska N, Midthune D, Potischman N, et al. (2011) Use of the predictive sugars biomarker to evaluate self-reported total sugars intake in the Observing Protein and Energy Nutrition (OPEN) study. Cancer Epidemiol Biomarkers Prev 20, 490-500.

52. Statens Jordbruksverk (2014) Livsmedelskonsumtion och näringsinnehåll/food consumption and nutritive values, data up to 2013. http://www.jordbruksverket.se/webdav/files/SJV/ Amnesomraden/Statistik,\%20fakta/Livsmedel/JO44SM1401/ JO44SM1401/JO44SM1401_tabeller16.htm (accessed May 2015).

53. National Food Agency (1999) Sweden National Food Consumption Survey 1997-1998. http://www.livsmedelsverket. se/globalassets/matvanor-halsa-miljo/kostrad-matvanor/ matvaneundersokningar/riksmaten-1997-1998-resultat-ochmetodrapport.pdf?_t_id=1B2M2Y8AsgTpgAmY7PhCfg\%3d\% 3d\&_t_q=Riksmaten+1997\&_t_tags=language\%3asv\%2csiteid \%3a67f9c486-281d-4765-ba72-ba3914739e3b\&_t_ip=130.235. 136.64\&_t_hit.id=Livs_Common_Model_MediaTypes_ DocumentFile/_f92fd7c3-7aa5-473a-969f-a7e293742c88\&_t_ hit.pos $=1$ (accessed November 2015). 